\title{
Numerical calculations of 2D transonic flow in GAMM channel and over the profile
}

\author{
Martin Slouka ${ }^{1, *}$, Karel Kozel ${ }^{1,2}$ \\ ${ }^{1}$ Dept. of Technical Mathematics, Faculty of Mechanical Eng., CTU in Prague, 12135 Prague 1, Czech Rep. \\ ${ }^{2}$ Institute of Thermomechanics, Academy of Science CR, 18200 Prague 8, Czech Rep.
}

\begin{abstract}
The aim of this work is to make a 2D numerical model of the solution of the transonic inviscid and viscous compressible flow around the profile. In a case of viscous flow several turbulent models are used. For the verification of the calculation Baldwin-Lomax model is compared with Wilcox k-omega model and SST turbulent model. Calculations are done in GAMM channel computational domain with $10 \%$ DCA profile and in the turbine cascade computational domain with $8 \%$ DCA profile. Numerical methods are based on a finite volume solution. Comparisons are done with the experimental data for the $8 \%$ DCA profile.
\end{abstract}

\section{Introduction}

Mathematical models used in this work are based on the solution of the two-dimensional transonic inviscid and viscous compressible flow. In a case of inviscid flow system of Euler equations is equipped. In a case of viscous flow, the simulation is based on the solution of the system of RANS equations. The system is completed by the algebraic Baldwin-Lomax model of turbulence, Wilcox k- $\omega$ model and the SST k- $\omega$ model.

Computational domain represents GAMM channel with $10 \%$ DCA profile and blade cascade with $8 \%$ DCA profile. The results show differences between the uses of each numerical model and experimental results.

\section{Governing equations}

The two-dimensional viscous turbulent compressible flow is prescribed by the system of RANS in the following conservation form

$$
\mathbf{W}_{\mathrm{t}}+\mathbf{F}_{\mathrm{x}}+\mathbf{G}_{\mathrm{y}}=\mathbf{R}_{\mathrm{x}}+\mathbf{S}_{\mathrm{x}}
$$

where

$$
\begin{gathered}
\mathbf{W}=(\rho, \rho u, \rho v, e)^{\mathrm{T}}, \\
\mathbf{F}=\left(\rho u, \rho u^{2}+p, \rho u v,(e+p) u\right)^{\mathrm{T}}, \\
\mathbf{G}=\left(\rho v, \rho u v, \rho v^{2}+p,(e+p) v\right)^{\mathrm{T}}, \\
\mathbf{R}=\left(0, \tau_{\mathrm{xx}}, \tau_{\mathrm{xy}}, u \tau_{\mathrm{xx}}+v \tau_{\mathrm{xy}}-q_{\mathrm{x}}\right)^{\mathrm{T}}, \\
\mathbf{S}=\left(0, \tau_{\mathrm{xy}}, \tau_{\mathrm{yy}}, u \tau_{\mathrm{xy}}+v \tau_{\mathrm{yy}}-q_{\mathrm{y}}\right)^{\mathrm{T}},
\end{gathered}
$$

$$
\begin{gathered}
\tau_{\mathrm{ij}}=\mu\left(\frac{\partial u_{\mathrm{i}}}{\partial x_{\mathrm{j}}}+\frac{\partial u_{\mathrm{j}}}{\partial x_{\mathrm{i}}}\right)-\frac{1}{3} \delta_{\mathrm{ij}} \frac{\partial u_{\mathrm{k}}}{\partial x_{\mathrm{k}}}, \\
q_{\mathrm{i}}=-\frac{\kappa}{\kappa-1} \frac{\mu}{\operatorname{Pr}} \frac{\partial}{\partial x_{\mathrm{i}}}\left(\frac{p}{\rho}\right) .
\end{gathered}
$$

This system is closed by following equation

$$
p=(\kappa-1)\left[e-\frac{1}{2} \rho\left(u^{2}+v^{2}\right)\right] \text {. }
$$

In the equations above $\rho$ denotes density, $(u, v)$ are components of local velocity in $x$ and $y$ direction respectively, $e$ denotes total energy per unit volume, $p$ is pressure given by previous equation of state, $\tau_{\mathrm{ij}}$ is shear stress, $q_{\mathrm{i}}$ represents heat flux and $\mu$ represents dynamical viscosity.

Following constants were used in previous equations: $\kappa$ is isentropic exponent equaled to $1.4, \mathrm{Pr}$ is Prandtl number equaled to the constant value 0.7 . Inviscid flow represented by the system of Euler equations is reached after neglecting of viscosity in the viscous fluxes on the right hand side in the system (1).

\section{Turbulence modeling}

Viscous compressible turbulent flow represents the system of Reynolds averaged Navier-Stokes equations (RANS) which is formally the same as (1) with the model of turbulence enclosed for the new variable of the turbulent viscosity. The equations from (7) and (8) are now modified as

and 


$$
\begin{gathered}
\tau_{\mathrm{ij}}=\left(\mu+\mu_{\mathrm{t}}\right)\left(\frac{\partial u_{\mathrm{i}}}{\partial x_{\mathrm{j}}}+\frac{\partial u_{\mathrm{j}}}{\partial x_{\mathrm{i}}}\right)-\frac{1}{3} \delta_{\mathrm{ij}} \frac{\partial u_{\mathrm{k}}}{\partial x_{\mathrm{k}}}, \\
q_{\mathrm{i}}=-\frac{\kappa}{\kappa-1}\left(\frac{\mu}{P r}+\frac{\mu_{t}}{P r_{\mathrm{t}}}\right) \frac{\partial}{\partial x_{\mathrm{i}}}\left(\frac{p}{\rho}\right),
\end{gathered}
$$

where $\mu_{\mathrm{t}}$ represents turbulent viscosity and $P r_{\mathrm{t}}$ is turbulent Prandtl number which is the constant value 0.9.

\subsection{Baldwin-Lomax model}

Baldwin-Lomax model [1] is the modification of basic Cebeci and Smith [2] algebraic turbulent model. The boundary layer is divided into two regions, inner region (close to the wall) and outer region. Turbulent viscosity in the inner part is given by

$$
\mu_{\mathrm{ti}}=\rho F_{\mathrm{d}}^{2} \kappa^{2} y^{2}|\Omega|
$$

where vorticity is in $2 \mathrm{D}$ case defined as

$$
\Omega=\frac{\partial u}{\partial y}-\frac{\partial v}{\partial x}
$$

van Driest function given as follow

$$
F_{\mathrm{d}}=1-\exp \left(-\frac{1}{A} \frac{u_{\tau} y}{v_{\mathrm{w}}}\right)
$$

and friction velocity by formula

$$
u_{\tau}=\sqrt{\frac{\tau_{\mathrm{w}}}{\rho}}=\left(v \frac{\partial u}{\partial y}\right)_{\mathrm{w}}^{1 / 2} .
$$

Turbulent viscosity in outer region is given by

$$
\mu_{\mathrm{to}}=\alpha C_{\mathrm{cp}} F_{\mathrm{w}} F_{\mathrm{k}} \text {. }
$$

Function $F_{\mathrm{w}}$ is determined by the relation

$$
F_{\mathrm{w}}=y_{\text {max }} F_{\text {max }}
$$

for the solid wall and by the relation

$$
F_{\mathrm{w}}=C_{\mathrm{wk}} y_{\text {max }} \frac{\Delta U^{2}}{F_{\text {max }}}
$$

for the wake after the profile. $F_{\max }$ is the maximum of the function

$$
F=y|\Omega| F_{\mathrm{d}}
$$

and $y_{\max }$ is the distance from the wall in which $F\left(\mathrm{y}_{\max }\right)=$ $F_{\max }$ holds and

$$
F_{\mathrm{k}}=\left[1+5.5\left(\mathrm{C}_{\mathrm{KL}} \frac{y}{y_{\max }}\right)^{6}\right]^{-1} .
$$

In Baldwin-Lomax turbulent model following constants are used:

$$
\begin{gathered}
\kappa=0.4, A=26, \alpha=0.0168, \\
C_{\mathrm{cp}}=1.6, C_{\mathrm{KL}}=0.3 \text { and } C_{\mathrm{wk}}=0.3 .
\end{gathered}
$$

\subsection{Wilcox k-w model (2006)}

The two-equation model is given by transport equations for two characteristic scales of turbulent motion, in this case turbulent energy $k$ and specific dissipation rate $\omega$. Generally, the turbulent viscosity is defined as follow

$$
\mu_{\mathrm{t}}=\rho \frac{k}{\omega} .
$$

The Wilcox k- $\omega$ model [3], [4] is given by the equations

$$
\begin{gathered}
\frac{\partial(\rho k)}{\partial t}+\frac{\partial\left(\rho u_{\mathrm{j}} k\right)}{\partial x_{\mathrm{j}}}= \\
P_{\mathrm{k}}+\frac{\partial}{\partial x_{\mathrm{j}}}\left[\left(\mu+\sigma^{*} \mu_{\mathrm{t}}\right) \frac{\partial k}{\partial x_{\mathrm{j}}}\right]-\beta^{*} \rho k \omega, \\
\frac{\partial(\rho \omega)}{\partial t}+\frac{\partial\left(\rho u_{\mathrm{j}} \omega\right)}{\partial x_{\mathrm{j}}}= \\
\gamma \frac{\omega}{k} P_{\mathrm{k}}+\frac{\partial}{\partial x_{\mathrm{j}}}\left[\left(\mu+\sigma \mu_{\mathrm{t}}\right) \frac{\partial \omega}{\partial x_{\mathrm{j}}}\right]-\beta \rho k \omega^{2}+C_{\mathrm{D}},
\end{gathered}
$$

where

$$
\begin{gathered}
C_{\mathrm{D}}=\sigma_{\mathrm{d}} \frac{\rho}{\omega} \max \left(\frac{\partial k}{\partial x_{\mathrm{j}}} \frac{\partial \omega}{\partial x_{\mathrm{j}}}, 0\right), \\
P_{\mathrm{k}}=\tau_{\mathrm{ij}} \frac{\partial u_{\mathrm{i}}}{\partial x_{\mathrm{j}}}, \\
\tau_{\mathrm{ij}}=\mu_{\mathrm{t}}\left(2 S_{\mathrm{ij}}-\frac{2}{3} \frac{\partial u_{\mathrm{k}}}{\partial x_{\mathrm{k}}} \delta_{\mathrm{ij}}\right)-\frac{2}{3} \rho k \delta_{\mathrm{ij}}, \\
S_{\mathrm{ij}}=\frac{1}{2}\left(\frac{\partial u_{\mathrm{i}}}{\partial x_{\mathrm{j}}}+\frac{\partial u_{\mathrm{j}}}{\partial x_{\mathrm{i}}}\right)
\end{gathered}
$$

and turbulent viscosity as given by

$$
\mu_{\mathrm{t}}=\rho \frac{k}{\hat{\omega}},
$$

where

$$
\begin{gathered}
\hat{\omega}=\max \left(\omega ; C_{\lim } \sqrt{\frac{2 \hat{S}_{\mathrm{ij}} \hat{S}_{\mathrm{ij}}}{\beta^{*}}}\right), \\
\hat{S}_{\mathrm{ij}}=S_{\mathrm{ij}}-\frac{1}{3} \frac{\partial u_{\mathrm{k}}}{\partial x_{\mathrm{k}}} \delta_{\mathrm{ij}} .
\end{gathered}
$$

The constants are:

$$
\begin{gathered}
\gamma=13 / 25, \beta_{0}=0.0708, \beta^{*}=9 / 100 \\
\sigma^{*}=3 / 5, \sigma=1 / 2 \text { and } C_{\lim }=7 / 8
\end{gathered}
$$

The auxiliary functions are:

$$
\begin{gathered}
\beta=\beta_{0} f_{\beta}, \\
f_{\beta}=\frac{1+85 \chi_{\omega}}{1+100 \chi_{\omega}}, \\
\chi_{\omega}=\left|\frac{\Omega_{\mathrm{ij}} \Omega_{\mathrm{jk}} \hat{S}_{\mathrm{ki}}}{\left(\beta^{*} \omega\right)^{3}}\right|, \\
\hat{S}_{\mathrm{ki}}=S_{\mathrm{ki}}-\frac{1}{2} \frac{\partial u_{\mathrm{m}}}{\partial x_{\mathrm{m}}} \delta_{\mathrm{ki}}, \\
\Omega_{\mathrm{ij}}=\frac{1}{2}\left(\frac{\partial u_{\mathrm{i}}}{\partial x_{\mathrm{j}}}-\frac{\partial u_{\mathrm{j}}}{\partial x_{\mathrm{i}}}\right) .
\end{gathered}
$$

\subsection{SST k-w model}

SST model [5] is combination of $\mathrm{k}-\omega$ and $\mathrm{k}-\varepsilon$ model in the following modification 


$$
\begin{aligned}
& \frac{\partial(\rho k)}{\partial t}+\frac{\partial\left(\rho u_{\mathrm{j}} k\right)}{\partial x_{\mathrm{j}}}= \\
& P_{\mathrm{k}}+\frac{\partial}{\partial x_{\mathrm{j}}}\left[\left(\mu+\sigma^{*} \mu_{\mathrm{t}}\right) \frac{\partial k}{\partial x_{\mathrm{j}}}\right]-\beta^{*} \rho k \omega, \\
& \frac{\partial(\rho \omega)}{\partial t}+\frac{\partial\left(\rho u_{\mathrm{j}} \omega\right)}{\partial x_{\mathrm{j}}}= \\
& \gamma \frac{\omega}{k} P_{\mathrm{k}}+\frac{\partial}{\partial x_{\mathrm{j}}}\left[\left(\mu+\sigma \mu_{\mathrm{t}}\right) \frac{\partial \omega}{\partial x_{\mathrm{j}}}\right]-\beta \rho k \omega^{2}+ \\
& 2 \rho\left(1-F_{1}\right) \sigma_{2} \frac{1}{\omega} \frac{\partial k}{\partial x_{\mathrm{j}}} \frac{\partial \omega}{\partial x_{\mathrm{j}}} .
\end{aligned}
$$

Turbulent viscosity is then given by

$$
\mu_{\mathrm{t}}=\min \left(\rho \frac{k}{\omega}, \rho \frac{a_{1} k}{F_{2} \Omega}\right),
$$

where $\Omega_{\mathrm{ij}}$ is given by (35) and functions $F_{1}$ and $F_{2}$ are defined as follow

$$
\begin{gathered}
F_{1}=\tanh \left(\Gamma_{1}^{4}\right), \\
\Gamma_{1}=\min \left[\max \left(\frac{\sqrt{k}}{\beta^{*} \omega y} ; \frac{500 v}{\omega y^{2}}\right) ; \frac{4 \rho \sigma_{2} k}{D_{\omega} y^{2}}\right], \\
D_{\omega}=\max \left(2 \rho \sigma_{2} \frac{1}{\omega} \frac{\partial k}{\partial x_{\mathrm{j}}} \frac{\partial \omega}{\partial x_{\mathrm{j}}} ; 10^{-20}\right) \\
F_{2}=\tanh \left(\Gamma_{2}^{2}\right) \\
\Gamma_{2}=\max \left(2 \frac{\sqrt{k}}{0,09 \omega y} ; \frac{500 v}{\omega y^{2}}\right) .
\end{gathered}
$$

Model constant are calculated according the following general formula

$$
\Pi=F_{1} \Pi_{1}+\left(1-F_{1}\right) \Pi_{2} .
$$

Constants of $\mathrm{k}-\omega$ model are:

$$
\begin{aligned}
& \beta_{1}=0.075, \sigma_{1}=0.5 \text { and } \\
& \gamma_{1}=\beta_{1} / \beta^{*}-\sigma_{1} \kappa^{2} / \sqrt{\beta^{*}} .
\end{aligned}
$$

Constant of $\mathrm{k}-\varepsilon$ model are:

$$
\begin{aligned}
& \beta_{2}=0.0828, \sigma_{2}=0.85 \text { and } \\
& \gamma_{2}=\beta_{2} / \beta^{*}-\sigma_{2} \kappa^{2} / \sqrt{\beta^{*}} .
\end{aligned}
$$

General constants for both are:

$$
\beta^{*}=0.09, \sigma^{*}=0.85, \kappa=0.41 \text { and } a_{1}=0.31 \text {. }
$$

\section{Numerical method}

\subsection{Numerical scheme}

A Finite volume method was applied on the cell centered non-orthogonal structured grid with quadrilateral cells.

For the numerical solutions Lax-Wendroff scheme in McCormack modification [6] was used with predictor step

$$
\mathbf{W}_{\mathrm{i}, \mathrm{j}}^{\mathrm{n}+1 / 2}=\mathbf{W}_{\mathrm{i}, \mathrm{j}}^{\mathrm{n}}-\Delta t \operatorname{Res} \mathbf{W}_{\mathrm{i}, \mathrm{j}}^{\mathrm{n}}
$$

and corrector step as follow

$$
\mathbf{W}_{\mathrm{i}, \mathrm{j}}^{\mathrm{n}+1}=\frac{1}{2}\left(\mathbf{W}_{\mathrm{i}, \mathrm{j}}^{\mathrm{n}}+\mathbf{W}_{\mathrm{i}, \mathrm{j}}^{\mathrm{n}+1 / 2}-\Delta t \operatorname{Res} \mathbf{W}_{\mathrm{i}, \mathrm{j}}^{\mathrm{n}+1 / 2}\right) .
$$

The Jameson's artificial dissipation model was applied to damp the oscillations.

$$
\begin{gathered}
\tilde{\mathbf{W}}_{\mathrm{i}, \mathrm{j}}^{\mathrm{n}+1}=\mathbf{W}_{\mathrm{i}, \mathrm{j}}^{\mathrm{n}+1}+A D\left(\mathbf{W}_{\mathrm{i}, \mathrm{j}}^{\mathrm{n}}\right), \\
A D\left(\mathbf{W}_{\mathrm{i}, \mathrm{j}}^{\mathrm{n}}\right)=C_{1} \psi_{1}\left(\mathbf{W}_{\mathrm{i}+1, \mathrm{j}}^{\mathrm{n}}-2 \mathbf{W}_{\mathrm{i}, \mathrm{j}}^{\mathrm{n}}+\mathbf{W}_{\mathrm{i}-1, \mathrm{j}}^{\mathrm{n}}\right)+ \\
C_{2} \psi_{2}\left(\mathbf{W}_{\mathrm{i}, \mathrm{j}-1}^{\mathrm{n}}-2 \mathbf{W}_{\mathrm{i}, \mathrm{j}}^{\mathrm{n}}+\mathbf{W}_{\mathrm{i}, \mathrm{j}-1}^{\mathrm{n}}\right)
\end{gathered}
$$

where the pressure correction functions are

$$
\begin{aligned}
& \psi_{1}=\frac{\left|p_{\mathrm{i}+1, \mathrm{j}}^{\mathrm{n}}-2 p_{\mathrm{i}, \mathrm{j}}^{\mathrm{n}}+p_{\mathrm{i}-1, \mathrm{j}}^{\mathrm{n}}\right|}{\left|p_{\mathrm{i}+1, \mathrm{j}}^{\mathrm{n}}\right|+\left|p_{\mathrm{i}, \mathrm{j}}^{\mathrm{n}}\right|+\left|p_{\mathrm{i}-1, \mathrm{j}}^{\mathrm{n}}\right|}, \\
& \psi_{2}=\frac{\left|p_{\mathrm{i}, \mathrm{j}+1}^{\mathrm{n}}-2 p_{\mathrm{i}, \mathrm{j}}^{\mathrm{n}}+p_{\mathrm{i}, \mathrm{j}-1}^{\mathrm{n}}\right|}{\left|p_{\mathrm{i}, \mathrm{j}+1}^{\mathrm{n}}\right|+\left|p_{\mathrm{i}, \mathrm{j}}^{\mathrm{n}}\right|+\left|p_{\mathrm{i}, \mathrm{j}-1}^{\mathrm{n}}\right|} .
\end{aligned}
$$

\subsection{Boundary conditions}

Inlet boundary conditions were realized for as follow: inlet velocity $M a_{\infty}$ together with angle of attack $\alpha$, density $\rho_{\infty}$ and total energy per volume $e_{\infty}$ were set; pressure $p_{\infty}$ was extrapolated from the flow field for the inlet Mach number $M a_{\infty}<1$, for the inlet Mach number $M a_{\infty} \geq 1$ pressure $p_{\infty}$ was set.

Outlet boundary conditions were done as follow: in a case of Mach number $M a_{\infty}<1$ outlet pressure was set and other variables were extrapolated from the flow field; in a case of Mach number $M a_{\infty} \geq 1$ all the variables were extrapolated from the flow field.

Solid wall condition was realized by the adding of virtual cells. In a case of inviscid flow velocity components were prescribed so that sum of velocity vectors equals zero in its tangential component. In a case of viscous flow, no-slip condition for the velocity was prescribed so that sum of velocity vectors equals zero on the wall.

Following parameters were chosen for the setting of turbulence models at the wall

$$
\begin{gathered}
k_{\mathrm{w}}=0, \\
\omega_{\mathrm{w}}=\frac{60 \mu}{\rho \beta y^{2}},
\end{gathered}
$$

and for the inlet flow

$$
\begin{gathered}
k_{\infty}=\frac{3}{2}\left(\operatorname{Int}_{\mathrm{T}} U_{\infty}\right)^{2}, \\
\omega_{\infty}=C_{\mu}^{-1 / 4} \frac{\sqrt{k}}{L},
\end{gathered}
$$

where the intensity of turbulence was prescribed as $\operatorname{Int}_{\mathrm{T}}=0.05$, constant $\mathrm{C}_{\mu}=0.09$ and dimension $L$ is a length of the used geometry.

\section{Numerical results}

The isolines of the Mach number are shown in all pictures. Flow parameters at the inlet are prescribed $M a_{\infty}$ corresponds to the inlet Mach number and $\alpha$ is an angle of the flow attack. Numerical results were chosen from a set of different setting of the inlet Mach number and angle of attack. Also the impact of the artificial dissipation model was checked to damp the oscillations 
and in a case of viscous model to eliminate the influence on the physical character.

First six figures correspond with the results of the calculations of transonic inviscid and viscous turbulent flow in the GAMM channel. This configuration was set as a reference for the testing of the behavior of both models. The calculation of the inviscid flow shows good result, the maximal value of Mach number $M a_{\max }=1.326$ is in comply with the expectation. Mach number along the profile is shown and have a good correlation with the result presented by Fürst [7]. The results of the viscous flow calculations have the good agreement to the each other, k- $\omega$ models shows the shock wave slightly higher than the algebraic model, maximal value of Mach number is around $M a_{\max }=1.166$ for the $\mathrm{k}-\omega$ models.

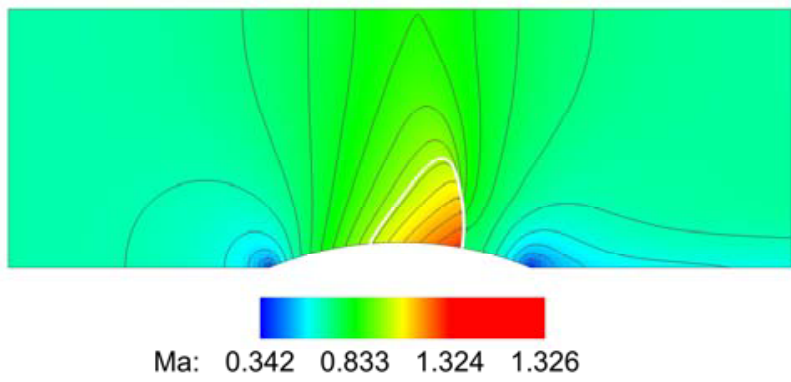

Fig. 1. GAMM channel, inviscid flow, $M a_{\infty}=0.675, \alpha=0.0^{\circ}$

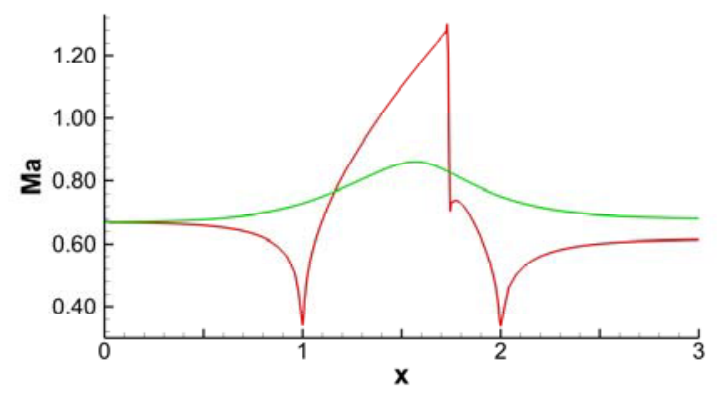

Fig. 2. GAMM channel, inviscid flow, $M a_{\infty}=0.675, \alpha=0.0^{\circ}$, distribution of Mach number along the profile

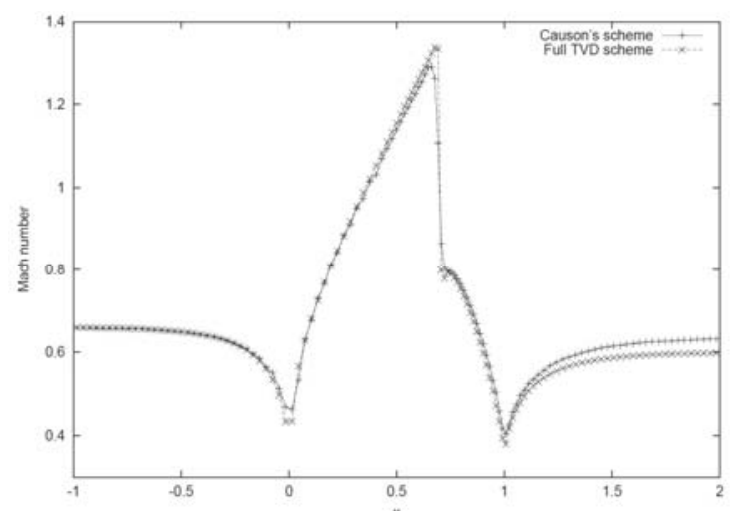

Fig. 3. GAMM channel, inviscid flow, $M a_{\infty}=0.675, \alpha=0.0^{\circ}$, distribution of Mach number along the profile, Fürst [7]

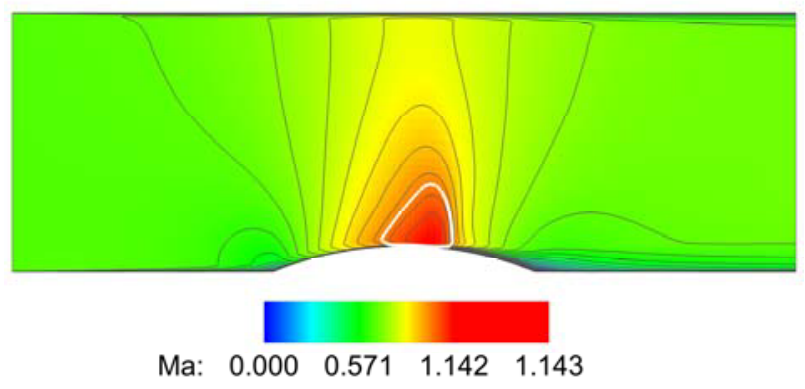

Fig. 4. GAMM channel, viscous turbulent flow, $M a_{\infty}=0.675$, $\alpha=0.0^{\circ}, \operatorname{Re}=10^{6}$, Baldwin-Lomax model

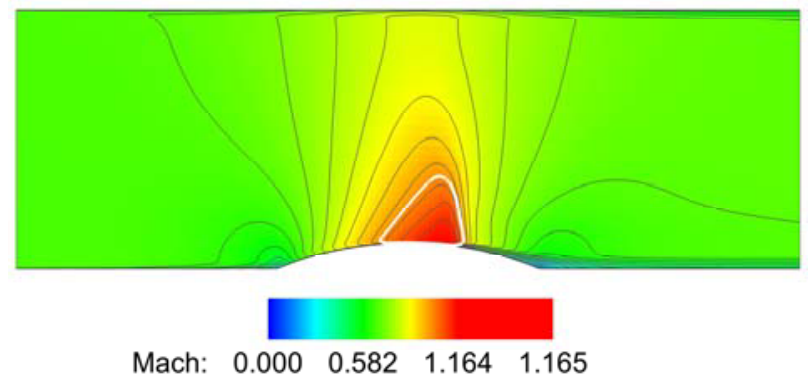

Fig. 5. GAMM channel, viscous turbulent flow, $M a_{\infty}=0.675$, $\alpha=0.0^{\circ}, \operatorname{Re}=10^{6}$, Wilcox $k$ - $\omega$ model

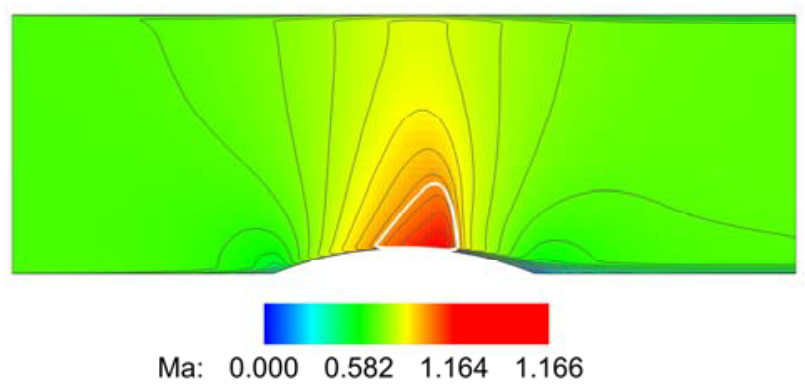

Fig. 6. GAMM channel, viscous turbulent flow, $M a_{\infty}=0.675$, $\alpha=0.0^{\circ}, \operatorname{Re}=10^{6}$, SST $k$ - $\omega$ model

The next set of figures shows the comparison between the used inviscid and viscous turbulent flow models and with experimental data of the measurements of the transonic flow through DCA cascade done by Institute of Thermomechanics AS CR in Prague and published in [8]. In this work there are shown the numerical results for the three different test cases and the comparison with the experimental data.

The inlet Mach number in the calculations was set slightly higher compare to the experiment. By the small change of the stream flow angle in the interval from $-3^{\circ}$ to $3^{\circ}$ the proper shape of the shock wave can be reached. These facts were considered in all calculations.

Figures 7 and 8 are corresponding with the experimental data shown on the figure 9. In a case of both numerical calculations the sonic line is visible on the upper profile and also on the lower profile although the sonic line in the experiment is only on the upper profile. 


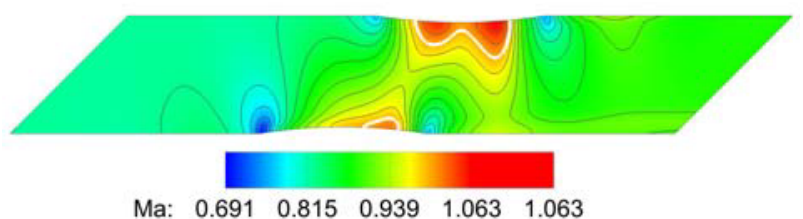

Fig. 7. DCA blade cascade, inviscid flow, $M a_{\infty}=0.830$, $\alpha=0.5^{\circ}$

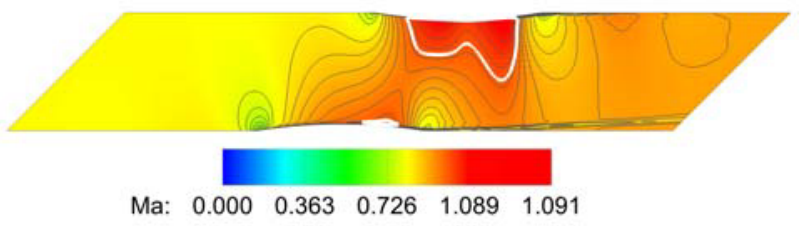

Fig. 8. $D C A$ blade cascade, viscous turbulent flow, $M a_{\infty}=0.835, \alpha=0.5^{\circ}, R e=10^{6}$, SST k- $\omega$ model

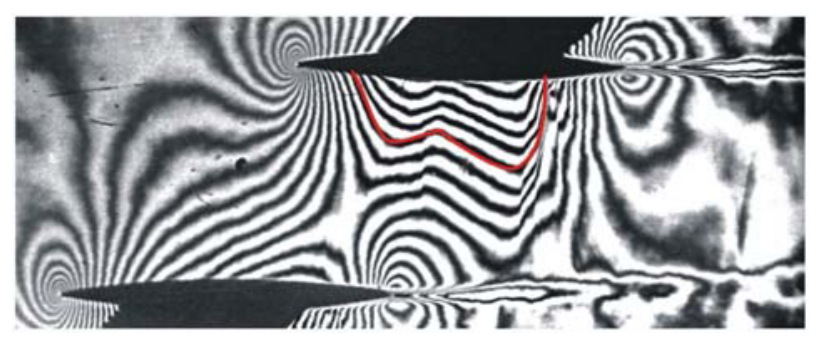

Fig. 9. Experimental data, $M a_{\infty}=0.813, \alpha=0.0^{\circ}$

Figures 10, 11 and 12 are corresponding with the experimental data shown on the figure 13 with the inlet Mach number $\mathrm{Ma}_{\infty}=0.946$. The position of the sonic line is similar for the all the numerical results in the upstream region and shows the good agreement with the upstream part of the experiment.

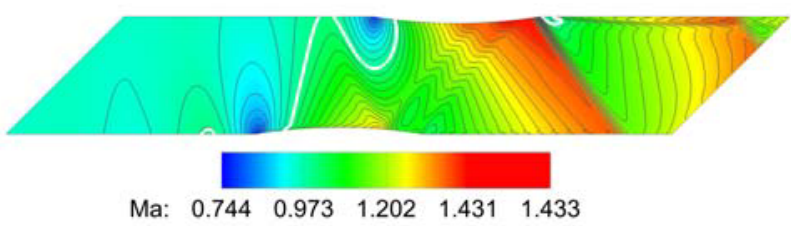

Fig. 10. DCA blade cascade, inviscid flow, $M a_{\infty}=0.980, \alpha=2.0^{\circ}$

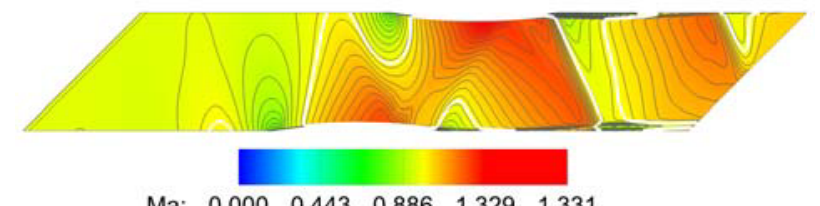

Fig. 11. DCA blade cascade, viscous turbulent flow, $M a_{\infty}=1.02, \alpha=0.0^{\circ}, \operatorname{Re}=10^{6}$, Wilcox $k-\omega$ model

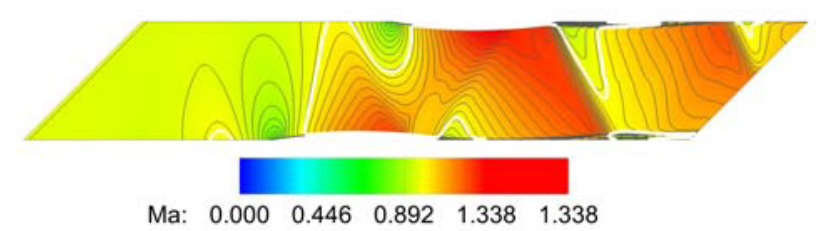

Fig. 12. DCA blade cascade, viscous turbulent flow, $M a_{\infty}=1.02, \alpha=0.0^{\circ}, \operatorname{Re}=10^{6}$, SST $k$ - $\omega$ model

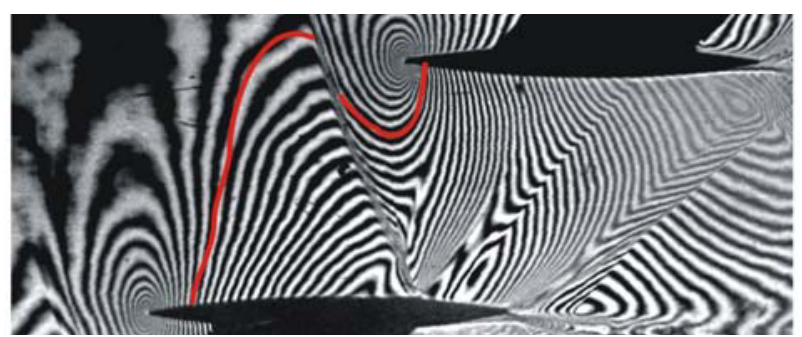

Fig. 13. Experimental data, $M a_{\infty}=0.946, \alpha=0.0^{\circ}$

The last set of the figures illustrates the comparison between the numerical results of the viscous flow calculations and the experimental data for the inlet Mach number $M a_{\infty}=1.073$. The sonic line is again presented in upstream and downstream part of the profiles and also the discontinuity in flow is visible. The quite good agreement with the experimental data is clear.

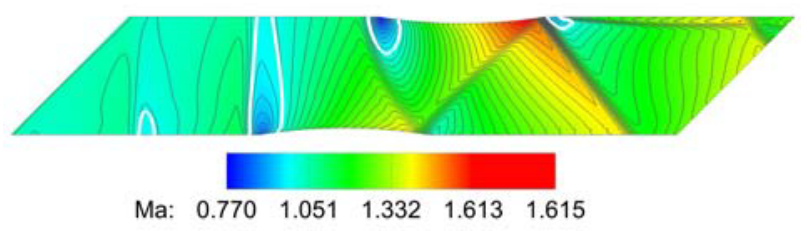

Fig. 14. $D C A$ blade cascade, inviscid flow, $M a_{\infty}=1.130$, $\alpha=0.0^{\circ}$

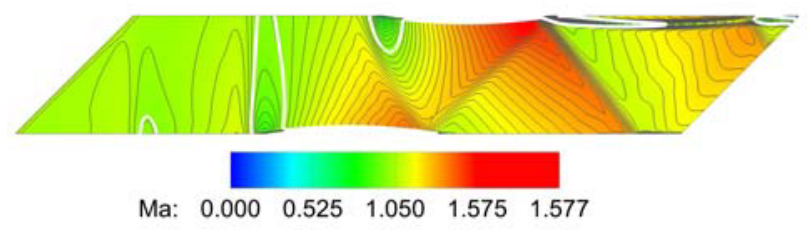

Fig. 15. DCA blade cascade, viscous turbulent flow, $M a_{\infty}=1.135, \alpha=0.0^{\circ}, R e=10^{6}$, Wilcox $k-\omega$ model

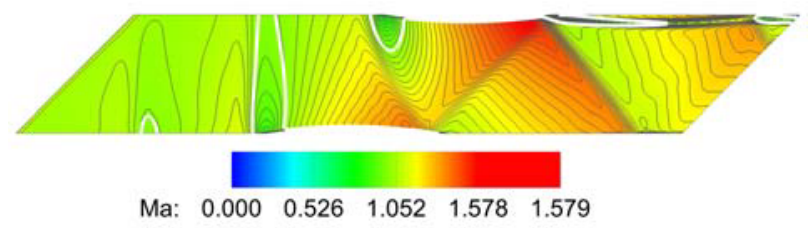

Fig. 16. DCA blade cascade, viscous turbulent flow, $M a_{\infty}=1.135, \alpha=0.0^{\circ}, \operatorname{Re}=10^{6}$, SST $k-\omega$ model

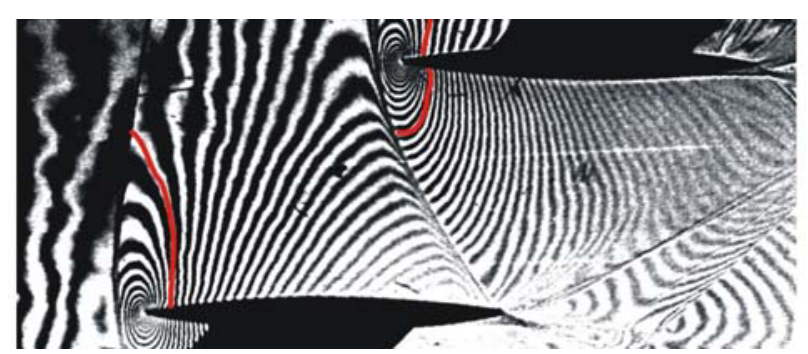

Fig. 17. Experimental data, $M a_{\infty}=1.073, \alpha=0.0^{\circ}$ 


\section{Conclusion}

The developed software was successfully tested for the calculations of inviscid and viscous turbulent flow in the GAMM channel and in the DCA blade cascade with the small region of the separation. The possibilities of different numerical approaches were described. The good correlation with the experimental data was shown. Achieved results are comparable with the other authors, e.g. [9], [10].

The research has been financed in part by grants $\mathrm{P}$ $101 / 10 / 1329$ and $P$ 101/12/1271 and by the Education for Competitiveness Operation Program.

\section{References}

1. B. Baldwin, H. Lomax, Thin layer approximation and algebraic model for separated turbulent flows, AIAA P., 78, 257 (1978)

2. T. Cebeci, A. M. O. Smith, Analysis of turbulent boundary layers, Acad. Press, New York (1974)

3. D. C. Wilcox, Turbulence modeling for CFD, DCW Ind., La Canada, Cal., 3rd edition (2006)

4. D. C. Wilcox, Formulation of the $k-\omega$ turbulence model revisited, AIAA J., 46, 2823-2838 (2008)

5. F. R. Menter, Two-equation eddy-viscosity turbulence models for engineering applications, AIAA J., 32, 1598-1605 (1994)

6. R. Dvorak, K. Kozel, Mathematical modelling in aerodynamics, CTU in Prague (1996)

7. J. Fürst, K. Kozel, Numerical solution of inviscid and viscous flows using modern schemes and quadrilateral or triangualar mesh, Math. Bohem., 2, 379-393 (2001)

8. R. Dvorak, Transonic flows, Academia, Prague, (1986)

9. J. Trefilik, K. Kozel, Proceedings: Recent advances in fluid mechanics and heat \& mass transfer, WSEAS, Florence, 193-196 (2011)

10. J. Trefilik, J. Huml, K. Kozel, J. Prihoda, Proceedings: Czech - Japanese seminar in applied mathematics 2010, CTU in Prague, 94-107 (2010) 